\title{
The DLR TuLam project: design of a short and medium range transport aircraft with forward swept NLF wing
}

\author{
A. Seitz ${ }^{1}\left(\mathbb{D} \cdot\right.$ A. Hübner ${ }^{1} \cdot$ K. Risse ${ }^{1}$
}

Received: 28 February 2018 / Revised: 30 August 2019 / Accepted: 9 September 2019 / Published online: 23 September 2019

(c) The Author(s) 2019

\begin{abstract}
In order to further raise the Technology Readiness Level (TRL) of laminar technologies in aircraft design the German Aerospace Center DLR conducted an internal project called TuLam (Toughen up Laminar Technology), which lasted from 2014 to 2017. In the course of the project two technology paths were pursued, namely Natural Laminar Flow (NLF) and Hybrid Laminar Flow Control (HLFC). Within the frame of the NLF path a short and medium range transport aircraft with forward swept laminar wing was designed. The present paper is focused on the aerodynamic design of the forward swept wing in cruise flight. As a special feature in comparison with previous designs of transonic laminar flow wings a trailing edge flap of $10 \%$ chord depth is employed to allow for an adaptation of the laminar bucket to off-design conditions. The resulting wing was assessed on overall aircraft level with respect to its fuel reduction potential, whereby the CSR-01 configuration, essentially a re-design of the Airbus A320-200, was used as a reference.
\end{abstract}

Keywords Short and medium range transport aircraft $\cdot$ Natural Laminar Flow $\cdot$ Airfoil design $\cdot$ Transonic wing

\section{Introduction}

Laminarization of boundary layers on wing, empennage and engine nacelles of transport aircraft has undoubtedly the highest potential for improving the aerodynamic performance. But before this technology can find its way into series production and become fully operational a bunch of scientific issues have to be resolved. For this reason the German Aerospace Center (DLR) has conducted the TuLam (Toughen up Laminar Technology) project in order to further raise the Technology Readiness Level (TRL), with the results of the predecessor project LamAiR (Laminar Aircraft Research, 2009-2012) as a starting point [1]. Like LamAiR, TuLam addresses the Natural Laminar Flow (NLF) and Hybrid Laminar Flow Control (HLFC) technology path.

Aerodynamics is inherently the key driver for development of laminar technology on transport aircraft. Consequently, throughout the past decades the basic problems regarding laminar to turbulent transition on transonic swept

A. Seitz

arne.seitz@dlr.de

1 DLR (German Aerospace Center), Institute of Aerodynamics and Flow Technology, Lilienthalplatz 7, 38108 Brunswick, Germany wings have been investigated thoroughly and flow control methodologies capable of delivering large portions of laminar boundary layer flow are well understood [2]. However, so far there are only a few fully developed laminar aircraft design studies available [3] that exceed conceptual or preliminary design level. But statements about performance gains resulting from laminarization that are obtained by conceptual or preliminary design methods are inevitably uncertain, because they rely on statistical and empirical approaches which are normally based on data of the numerous existing full chord turbulent wing concepts. Therefore, it is often not possible to model all the specific needs that come along with laminar technology and that might affect also other disciplines like structures.

In order to overcome this lack of information and to further build up a data base for the design of laminar aircraft the TuLam project pursued the detailed design of two laminar wetted aircraft components. Target application in both cases was a new short and medium range transport aircraft with the Top Level Aircraft Requirements of an A320-200, which has also been taken as a reference for the performance evaluation.

In the HLFC-path a fin (or vertical tailplane) with pressure distributions optimized for extensive laminar flow 
and equipped with an innovative tailored skin single duct (TSSD) suction system [4] was further developed.

Work in the NLF-path, described in the current paper, was dedicated to the aerodynamic design of a laminar wing. In the following sections, first the forward (or negative) sweep is motivated and then the aircraft configuration including the high-lift system is defined. Based on the configuration definition a sizing with a preliminary design method was performed that delivered the basic technical data of the aircraft, especially the main dimensions of the mono-trapezoidal wing.

With the data coming from the overall aircraft sizing as an input, an inverse design of two generator airfoil sections utilizing a $2.75 \mathrm{~d}$ RANS method was done. In order to enhance the off-design behavior of the wing sections, especially with respect to robust laminar flow, the beneficial effect of a $10 \%$ chord trailing edge flap was investigated. The $3 \mathrm{~d}$ wing generated from the sections, showed potential for improvement over the inboard wing, i.e. from the root to about $30 \%$ half span. Consequently, this area was redesigned employing the $3 \mathrm{~d}$ invers capabilities of the RANS code. Work on the detailed aerodynamic design is described in Sect. 4 of this paper.

Using the data from the RANS analysis of the wing-body combination, a drag estimation was performed for the forward swept laminar wing and used as a basis for the assessment of block fuel reductions on overall aircraft level for two typical mission profiles of the short and medium range transport. These results are presented and discussed in Sect. 5. A summary with concluding remarks and prospects for further work will close the paper.

\section{The TuLam configuration}

\subsection{Why negative sweep?}

In order to attenuate compressibility effects, wings of modern transport aircraft flying in the transonic flow regime (but still at subsonic free stream Mach numbers) are swept. Because of the leading edge sweep the flow will not come to complete stagnation but an attachment line will be formed that already develops a boundary layer. This boundary layer is prone to laminar to turbulent transition which inevitably will lead to a subsequently full chord turbulent flow. Occurrence of the phenomenon, well known as attachment line transition (ALT), will be more likely with increasing leading edge sweep.

If ALT has successfully been avoided, a three-dimensional boundary layer is formed downstream of the attachment line which exhibits a so called crossflow velocity profile with at least one inflectional point. Such velocity profiles are inherently unstable against small disturbances and the growth rate of these so-called crossflow instabilities (CFI) again depends on the local sweep. The higher the sweep, the more intense the crossflow and the stronger the amplification of CFI will be, leading to premature transition in the nose region of the wing.

For both these reasons it is desirable to have a leading edge sweep as low as possible, while, on the other hand, sweep in the recompression zone of the wing at about $50-60 \%$ of chord, where the shock is located, should be as high as possible. Not only will this allow for extensive laminar boundary layer flow but it will also keep the wave drag, which is associated with the shock, low. Both measures can be achieved if a forward sweep of the wing is chosen. Figure 1 than shows schematically, how in conjunction with taper (always necessary for low induced drag) the favorable increase of sweep in chordwise direction can be adjusted.

\subsection{Static aeroelasticity of the forward swept wing}

One drawback of the forward swept wing concept is its static aeroelasticity: In cases where isotropic materials, for example aluminum, are used for construction, an upward bending of the wing produces in all sections that are in line of flight an increase in angle of attack, which, in turn, leads to additional lift forces. Subsequently, static divergence with structural failure is possible, [5].

But this phenomenon can be avoided by a structural layout of the wing box employing fiber composites. The anisotropic characteristics of carbon fiber reinforced plastics (CFRP) allows for a coupling of flexional and torsional deformation of wings. This is achieved by an appropriate layer structure with variation of fiber direction that will lead to a derotation of wing sections parallel to the oncoming flow in case of an upward bending. This technology, well known as Aeroelastic Tailoring, was already applied during

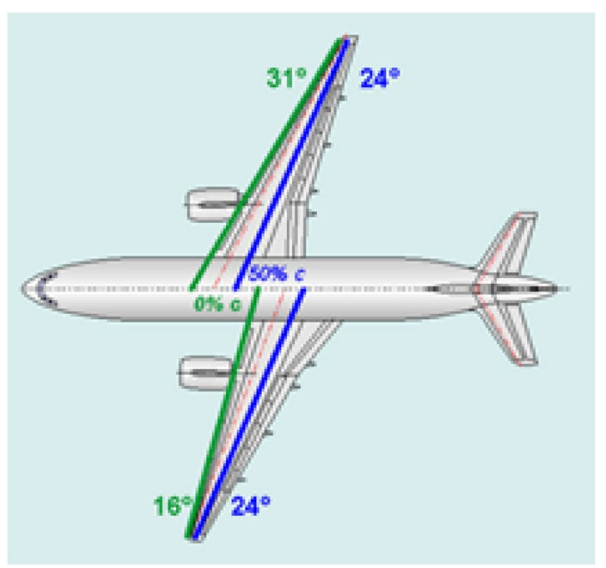

Fig. 1 Sweep angle at leading edge and at 50\% chord of a forward and a backward swept wing 
the LamAiR project to perform an aero-structure-coupled wing design (see Fig. 2 and [6]) with high-fidelity methods. By this measure, a wing was realized with a structural mass not higher than for a backward swept wing of conventional construction. Hence, for the TuLam project presented here it was assumed that the aeroelastic problem is basically resolved and the structural modeling in the preliminary design methods used here was updated accordingly.

\subsection{The target configuration}

Based on the above explanations the target configuration for the TuLam project was defined. Herein not only the important results from the LamAiR project were taken into consideration but also those from the EU-project DeSiReh (Design, Simulation and Flight Reynolds Number Testing for Advanced High-Lift Solutions). Figure 3 shows the resulting concept of the short and medium range aircraft, which, besides the forward swept laminar wing, has the following features:

1. The wing planform is a mono trapezoid with $17^{\circ}$ sweep at the leading and $28^{\circ}$ at the trailing edge. In the LamAiR project, these sweep angles had been shown to be the optimum for controlling of ALT and CFI while at the same time shock strength is moderate.

2. Results of the already mentioned aero-structure-coupled wing design performed in LamAiR [6] showed that for the chosen mono trapezoid a slightly non-elliptic spanwise wing loading with higher loads on the inner wing and thus reduced wing root bending moment delivers the lighter wing and, although aerodynamically (with respect to induced drag of the wing) not the optimum, the more efficient aircraft on Overall Aircraft Design (OAD) level. Therefore, this wing loading was prescribed also as target for the TuLam configuration. Additionally, the spanwise distribution of relative air-

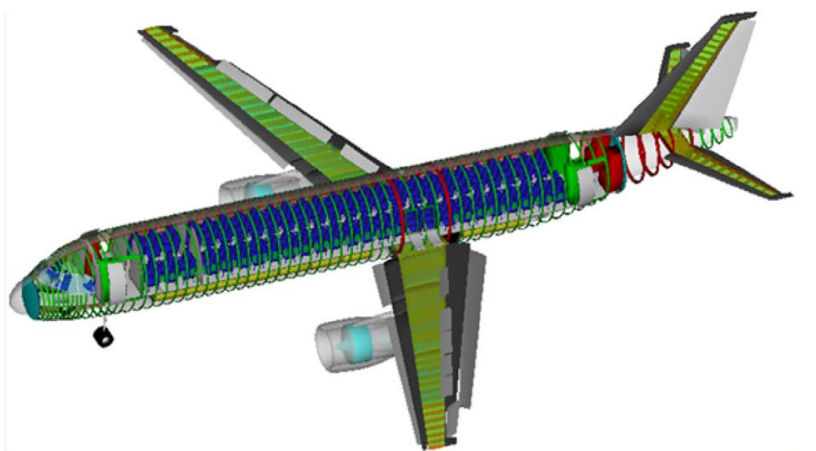

\begin{tabular}{|l|l|l|}
\hline & Notation & \\
\hline wing area & $\mathrm{S}$ & $122.0 \mathrm{~m}^{2}$ \\
\hline wing span & $\mathrm{b}$ & $34.0 \mathrm{~m}$ \\
\hline aspect ratio & $\Lambda$ & 9.4754 \\
\hline taper ratio & $\lambda$ & 0.3402 \\
\hline chord at tip & $\mathrm{C}_{\mathrm{t}}$ & $5.08002 \mathrm{~m}$ \\
\hline chord at root & $\mathrm{C}_{\mathrm{r}}$ & $1.72814 \mathrm{~m}$ \\
\hline mean aerodynamic chord & $\mathrm{C}_{\mathrm{MAC}}$ & $3.896 \mathrm{~m}$ \\
\hline sweep at leading edge & $\varphi_{\mathrm{le}}$ & $-17.0^{\circ}$ \\
\hline sweep at trailing edge & $\varphi_{\mathrm{te}}$ & $-27.8^{\circ}$ \\
\hline sweep at 60\% chord & $\varphi_{60}$ & $-23.6^{\circ}$ \\
\hline dihedral & $\boldsymbol{v}$ & $4.0^{\circ}$ \\
\hline front spar position & $(\mathrm{x} / \mathrm{c})_{\mathrm{fs}}$ & 0.17 \\
\hline rear spar position & $(\mathrm{x} / \mathrm{c})_{\mathrm{rs}}$ & 0.65 \\
\hline
\end{tabular}

Fig. 3 The TuLam target configuration with the main dimensions of the wing

foil thicknesses $t / c$ achieved in LamAiR was used for orientation (see also Fig. 9).

3. The engines are mounted in an underwing position. This installation delivers the more efficient aircraft, because
Fig. 2 Aero-structure coupled design of the LamAiR configuration; bending and torsional deformation of the forward swept wing at $\eta=0.9$ under load, [6]

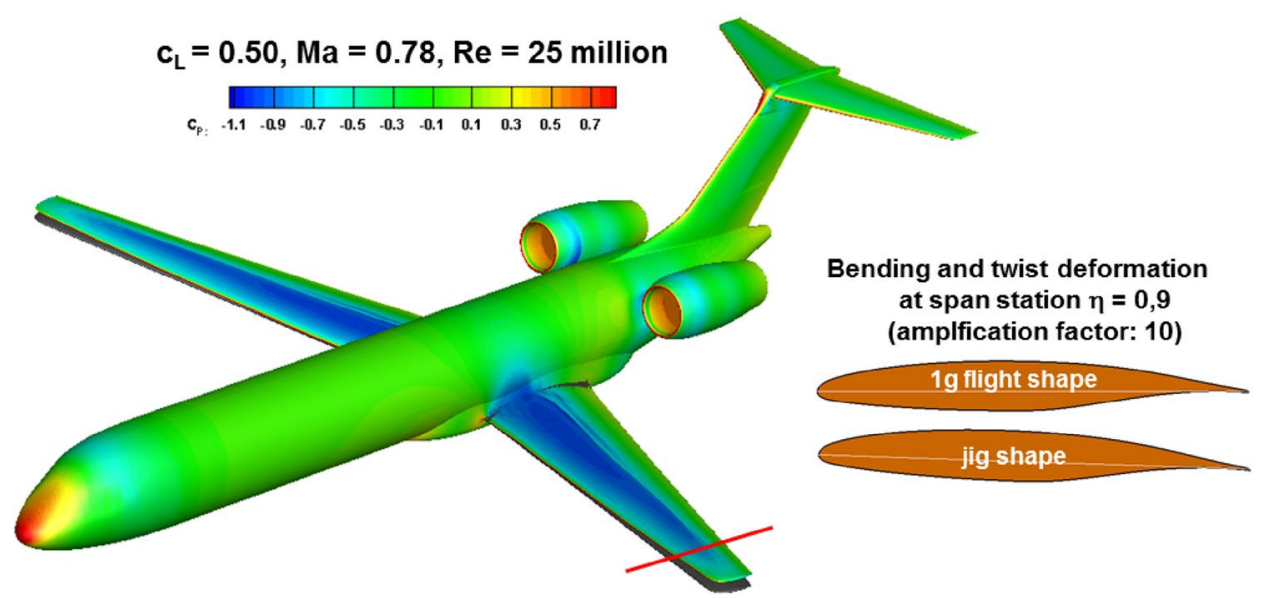


the rear position (like in LamAiR) will lead to a heavier fuselage structure, an effect which is even worsened by a comparatively heavy T-tail.

4. In LamAiR a so-called "Smart Droop Nose" was planned as leading edge high-lift device, which, in retracted position, exhibits a smooth and seamless surface across the joint of wing nose and box. Therefore, such a device is compatible with laminar flow on upper and lower surface, but the gain in maximum lift is limited. Hence, a comparatively large wing area $\left(132 \mathrm{~m}^{2}\right)$ was necessary to match landing performance requirements. For the TuLam configuration the concept of a "Bull-Nose-Krüger" was chosen (see Fig. 4 and [7]). However, a Krüger has the disadvantage of allowing laminar flow only on the upper surface, while transition on the lower surface is inevitably triggered by steps and gaps that are present when retracted. But this is compensated by higher maximum lift capabilities and, hence, a smaller wing area. Furthermore, the Krüger has a shielding effect, i.e. it protects the leading edge from being contaminated by insects hitting the wing during take-off or landing. As trailing edge device, a Fixed Vane Fowler flap is used.

5. For the sake of honest performance comparisons, dimensions of fuselage and empennage are equivalent to those of the A320-200. As an option, the laminar fin developed in the HLFC path of TuLam is foreseen for utilization.

6. Wing span is $34 \mathrm{~m}(\mathrm{~A} 320-200: 34.1 \mathrm{~m})$.

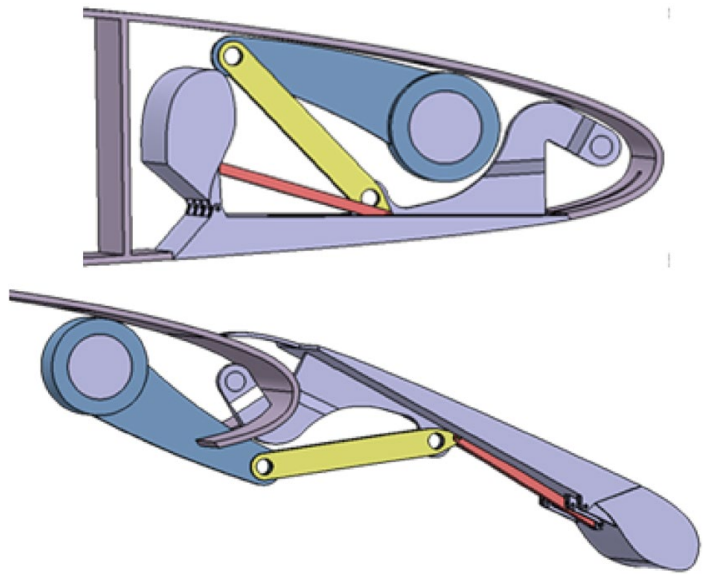

Fig. 4 Concept of a Bull Nose Krüger as a high-lift device (EU-project DeSiReh, [7])

\section{Preliminary design}

For the TuLam target configuration defined in Sect. 2.3 a sizing was performed employing the preliminary design method PrADO developed by TU Braunschweig, [8]. The goal was to obtain the main technical data which are used as an input for the detailed aerodynamic design of the wing. Because the A320-200 shall serve as a reference for TuLam, the "Top Level Aircraft Requirements (TLAR)" of this aircraft have been applied also here:

1. The payload range diagram with a design mission of $2500 \mathrm{NM}$ range and a payload of 17t (150 PAX plus cargo).

2. The design cruise Mach number is $\mathrm{Ma}=0.78$.

3. Take-off and landing distance shall not exceed $1900 \mathrm{~m}$ and $1470 \mathrm{~m}$, respectively, at the allowable maximum weights (MTOW and MLW).

Figure 3 shows as a result of the preliminary design the final TuLam configuration as well as a table containing the main dimensions of the forward swept wing. Beneath these data, from the profile of the design mission and the projected flight envelope, Fig. 5, the free stream conditions and the lift requirements are derived as an input for the subsequent design of generator airfoil sections and the forward swept wing.

\section{Detailed aerodynamic design}

\subsection{Airfoil design}

As design point for the generator airfoil sections of the forward swept laminar wing the center of the cruise domain in the flight envelope was defined, Fig. 5. Accordingly, the freestream Mach number is $\mathrm{Ma}=0.78$ and the flight level 35,000ft, leading to a chord Reynolds number of $\mathrm{Re}_{\mathrm{AMC}}=24$ million, while the average total lift coefficient during the design mission comes to $C_{\mathrm{L}}=0.52$.

In a first step, two generator sections were designed to be fitted into the wing at dimensionless span stations $\eta=0.294$ and $\eta=0.56$, where Reynolds number and required lift coefficient were adapted to the local conditions (i.e. scaling of Re via the local chord, while the local lift coefficient $\mathrm{cl}$ is taken from the spanwise lift distribution of the preliminary aircraft design). As a further geometrical constraint the required relative maximum thickness of both sections was prescribed in accordance with the LamAiR results, Fig. 9, to be $t / c=13.0 \%$ at $\eta=0.294$ and $t / c=11.5 \%$ at $\eta=0.560$. 
Fig. 5 Projected flight envelope of the TuLam configuration

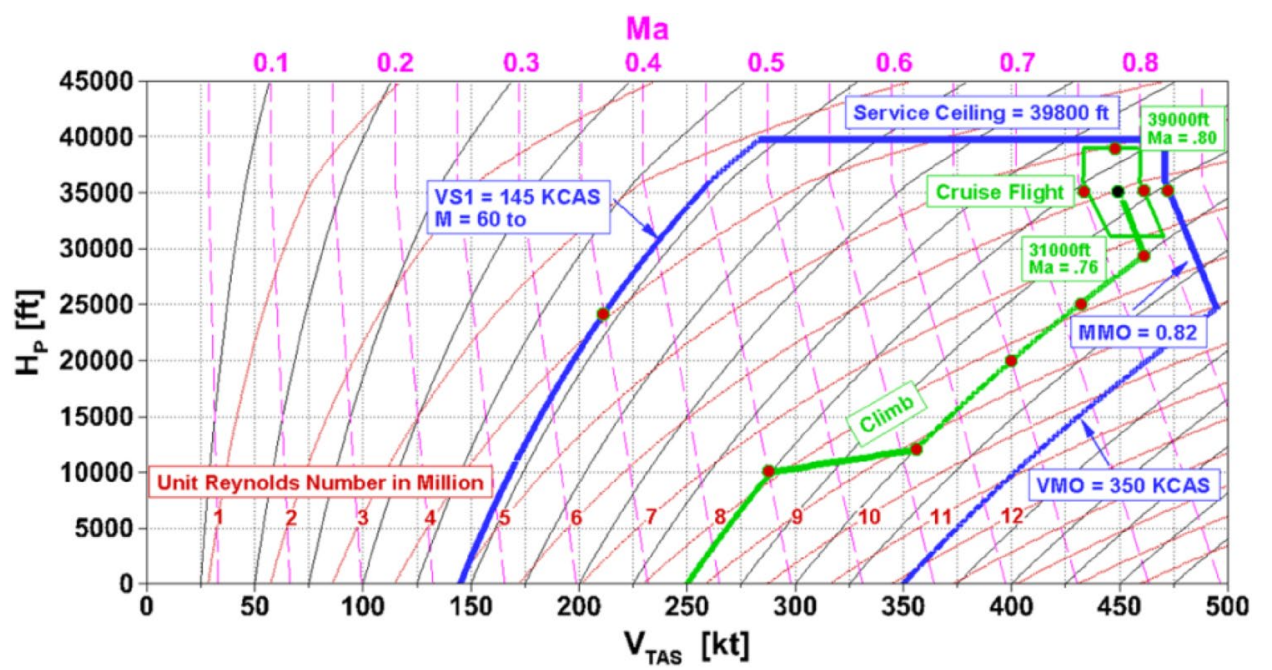

Utilizing the $2.75 \mathrm{~d}$ FLOWer method developed by Streit [9], the design was conducted inversely, i.e. the geometry of airfoil sections in line of flight are calculated iteratively until a prescribed target pressure distribution is matched, whereby effects of sweep and taper are taken into account.

Results of the airfoil design step are presented in Fig. 6. Shown are pressure distribution and geometry of generator sections at span stations $\eta=0.294$ (bottom) and $\eta=0.560$ (top). On the upper surface the pressure follows the typical sequence of transonic laminar airfoils. In the same diagram, $\mathrm{N}$-factor distributions can be found showing the amplitude growth of crossflow $\left(N_{\mathrm{CF}}\right)$ and Tollmien-Schlichting instabilities $\left(N_{\mathrm{TS}}\right)$. Amplitudes of both families of disturbances are kept low over the whole laminar run of the upper surface boundary layer and are well below the limit, which is at about $N=9-10$. Transition then occurs beyond the pressure minimum at about $60 \%$ of chord due to a strong growth of $N_{\mathrm{TS}}$, which follows the pressure rise that comes along with the compression shock at the same position. Drag estimation for this laminar airfoil delivers a coefficient of $c_{\mathrm{d}}=47 \mathrm{dc}$ (drag count) incorporating a wave drag of only $1 \mathrm{dc}$ (for comparison: a typical full chord turbulent profile with about the same thickness and lift requirements at comparable Reynolds number would have a drag in the order of $c_{\mathrm{d}}=80$ $-88 \mathrm{dc}$, whereof about $10 \mathrm{dc}$ wave drag).

Because of the usage of a Krüger as leading edge device and thus an inevitably early transition due to gaps and steps, a laminar pressure distribution is not necessary on the lower side of the wing. Therefore it was decided to realize a front loading there. By this measure it was possible to match the lift requirement of $c_{1}=0.582$ with a moderate rear loading. This has two advantages: Firstly, the nose down pitching moment coefficient can be kept low and, secondly, the recompression on the upper surface behind the shock can be designed with a moderate gradient that even relaxes towards the trailing edge. Such type of moderate pressure rise (roughly following the idea of Stratford) limits the growth of momentum loss thickness of the turbulent boundary layer on the upper rear part of the profile. It is well known from subsonic airfoil design, Eppler [10], that this local measure contributes to a low overall airfoil drag. However, with a Stratford type of pressure distribution stall may occur abruptly because the boundary layer on the upper surface is, for high angle of attack, close to separation at each chordwise position $x / c$ from leading edge to trailing edge.

For the present work, stall characteristics (at low speed) or buffet onset (at high speed) of the designed airfoil sections were not investigated in detail, but a quick look on basis of pressure distributions calculated with the $2.75 \mathrm{~d}$ FLOWer code was done at two points of the flight envelope in order to check if the flow at these operating points is free from separation (low speed flight at VS1 in 24,000ft and high speed flight at MMO of 0.82 in 35,000ft). Although these checks indicated no separation, a statement with higher level of confidence can only be given using hifi numerical analysis methods for the final, complete aircraft configuration. However, the ultimate proof as well as an assessment of stall behavior has to be done by an experimental investigation.

For other points of the flight envelope (red dots in Fig. 5) the questions arose if still a drag reduction gain by laminarization can be expected. As an example, Fig. 7 shows the result of the aerodynamic analysis for the left boundary of the cruise flight domain for the section at $\eta=0.560$. At a reduced free stream Mach number of $\mathrm{Ma}=0.76$ and constant total lift force the calculation was conducted for a slightly higher local lift coefficient of $c_{1}=0.613$. One can recognize that the pressure distribution on the suction side has been filled up in the front region by an increase in angle of attack. This leads to a reduced pressure gradient which, in turn, has a negative impact on the growth of Tollmien-Schlichting instabilities. In comparison to the design point, $N_{\text {TS }}$ reaches its critical value already at $42 \%$ of chord. Although there still 

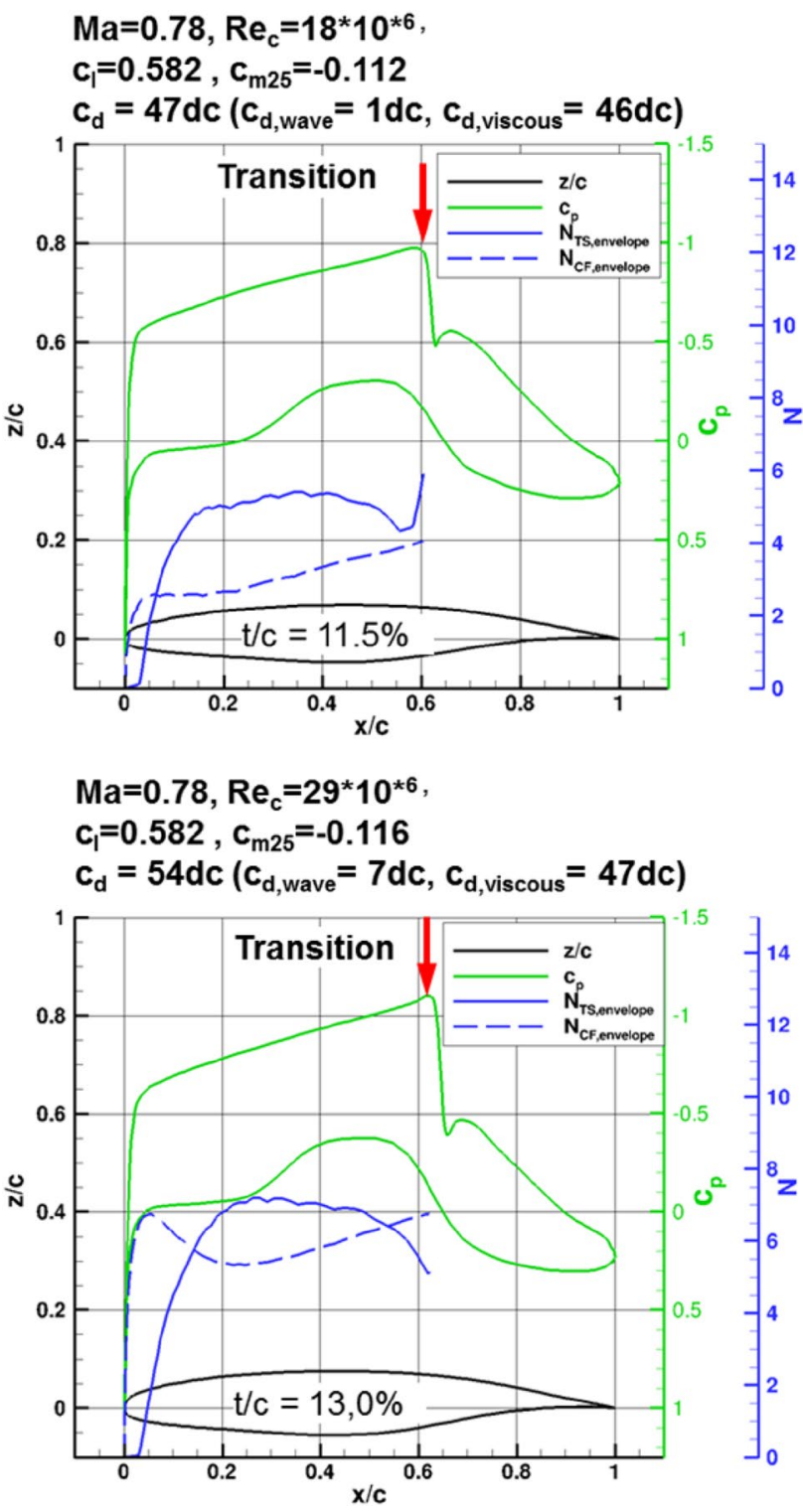

Fig. 6 Pressure and $\mathrm{N}$-factor distributions together with integral coefficients of generator wing sections at $\eta=0.294$ (bottom) and $\eta=0.560$ (top)

is a drag benefit compared to a full chord turbulent design, the gain is already reduced and, even worse, the $N_{\mathrm{TS}}$ distribution shown implies a rapid movement of transition in direction of the leading edge if the angle of attack is increased only a bit more. It is obvious that, under these conditions, the airfoil is operated at the edge of its laminar bucket in the drag polar.

As a simple measure to improve the off-design behavior, a trailing edge flap of $10 \%$ chord was introduced and its effect investigated. The result is shown in the right hand part of Fig. 7 for the $11 \%$ thick airfoil at $\eta=0.560$ : A downward flap deflection of $+5^{\circ}$ fixes the problem by increasing camber and, hence, the rear loading. This is possible because of the initially moderate pressure rise on the upper rear at flap deflection $0^{\circ}$ that came along with the Stratford type of pressure distribution. As can be seen on the right hand side of Fig. 7, the favorable gradient on the front part of the profile has been regained while $N_{\text {TS }}$ could be lowered sufficiently.

Further calculations are indicating that this effect can also be expected for operating points in climb, whereby here, with appropriate flap deflections, increasing flight altitude and therefore increasing Mach number but decreasing Reynolds number the wing will become laminar step by step from tip to root. However, this hypothesis has to be proven for the final wing design in detailed $3 \mathrm{~d}$ hifi calculations.

\subsection{Wing design}

By the definition of the TuLam target configuration and the preliminary design step some of the classical wing design work has already been completed, i.e. wing planform, lift and twist distribution are determined. With the previously designed airfoil sections the mono-trapezoidal wing shown in Fig. 8 was generated. The $11.5 \%$ thick airfoil is located at the wing tip and $\eta=0.560$ (pos. 5), while the $13.0 \%$ thick airfoil can be found at $\eta=0.294$ (pos. 4). Root section, belly fairing and fuselage were taken from the LamAiR project. Between the generator sections the wing was linearly interpolated. The result of the aerodynamic analysis of the wingbody-combination at the above defined design point with the structured RANS solver FLOWer is shown on the left of Fig. 8. It can be seen, that pressure distributions at pos. 4 and 5 meet the $2.75 \mathrm{~d}$ design. Additionally, isobars are straight lines that coincide with constant percent lines even at the shock location. But when approaching the root via pos. 3, 2 and 1 isobars tend to become more and more curved lines with the sweep at the shock gradually diminishing. This "center effect" results from the fact that the fuselage acts on the flow as a symmetry plane and the intersection of isobars and fuselage can only be perpendicular. As a consequence, the compression shock is no longer oblique but straight with a corresponding increase in local wave drag.

Obviously, a purely geometrical sweep of the wing is not sufficient in the region close to the fuselage. In fact, additionally aerodynamic design measures have to be applied in order to adapt the wing sections in a way that the isobars coincide with constant percent lines as far as possible. In the present case, the $3 \mathrm{~d}$ inverse design capabilities of the FLOWer code were used to meet that goal. The result is presented on the right hand side of Fig. 8. Because a turbulent wedge will emanate from the intersection of wing leading edge and fuselage anyway, a conventional roof-top like pressure distribution was prescribed at pos. 1 which gradually changes via pos. 2 over to the laminar distributions at pos. 3 and 4. As expected, the airfoil sections generated for spanwise positions close to the root are rather thick (e.g. 
$M a=0.76, R e=17^{\star} 10^{\star 6}\left(A / C C_{L}=0.547\right.$, constant lift, section $\left.c_{l}=0.613\right)$ +5 deg flap deflection shifts transition location from $42 \%$ to $58 \%$ chord which reduces profile drag from $c_{d}=58 d c$ down to $47 \mathrm{dc}$

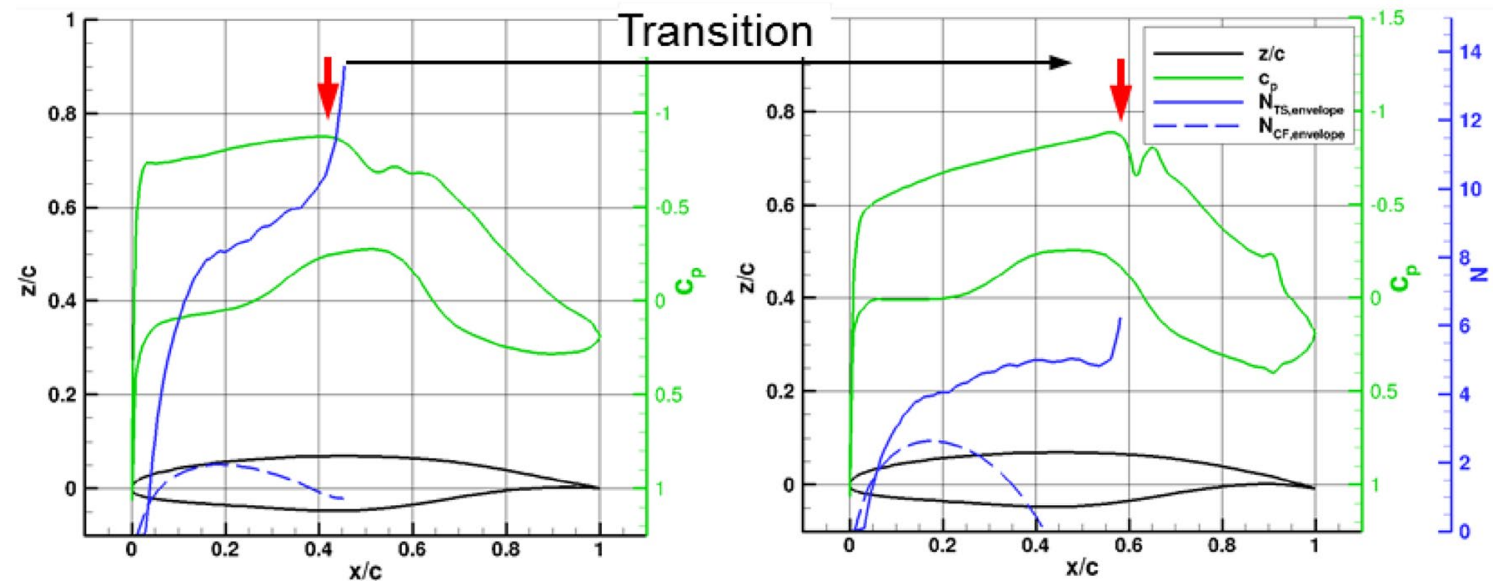

Fig. 7 Effect of a trailing edge flap that enhances the off-design drag characteristics of a laminar airfoil

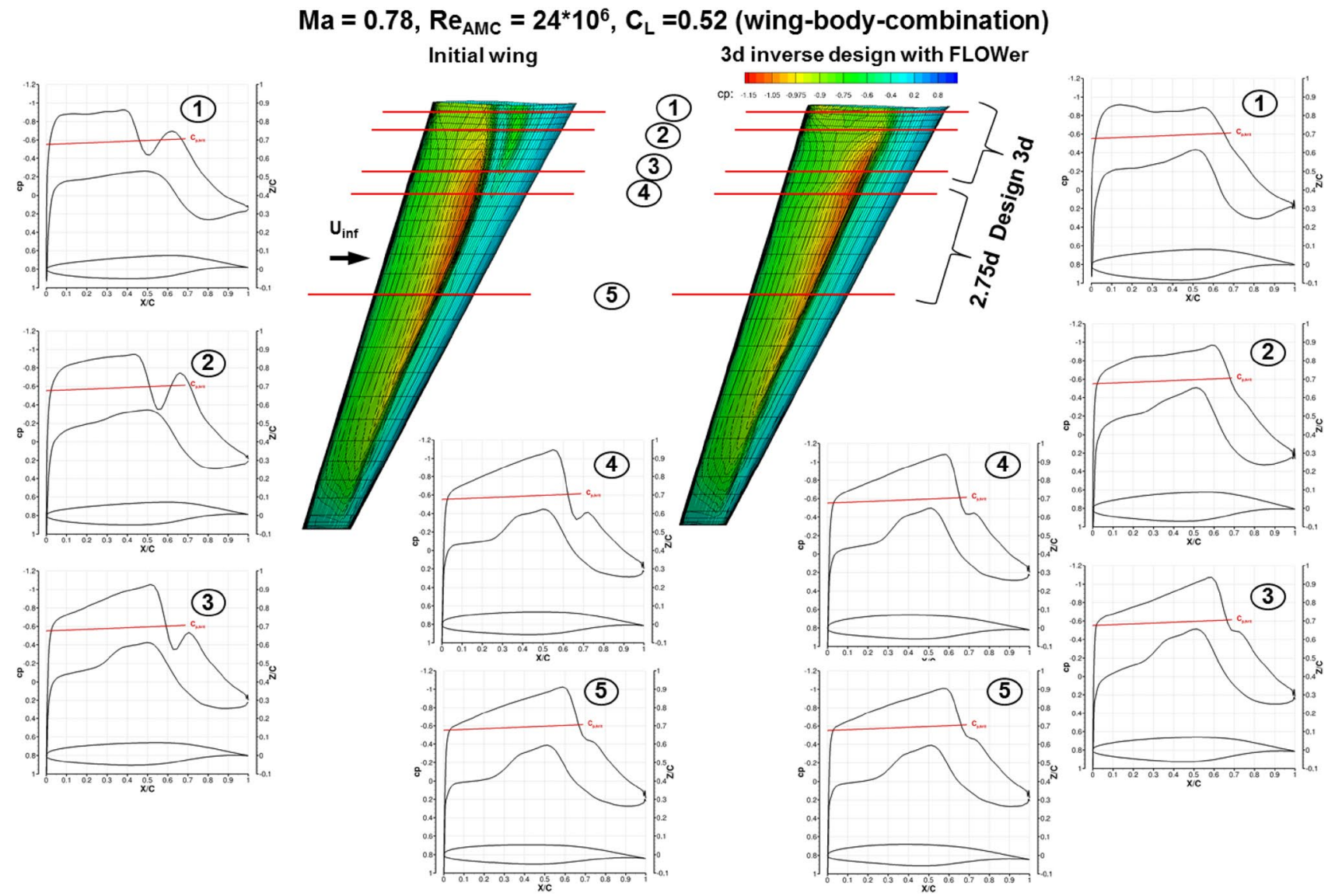

Fig. 8 TuLam 3d wing design; left: starting point; right: final design; shown are the isobars on the suction side of the wing 


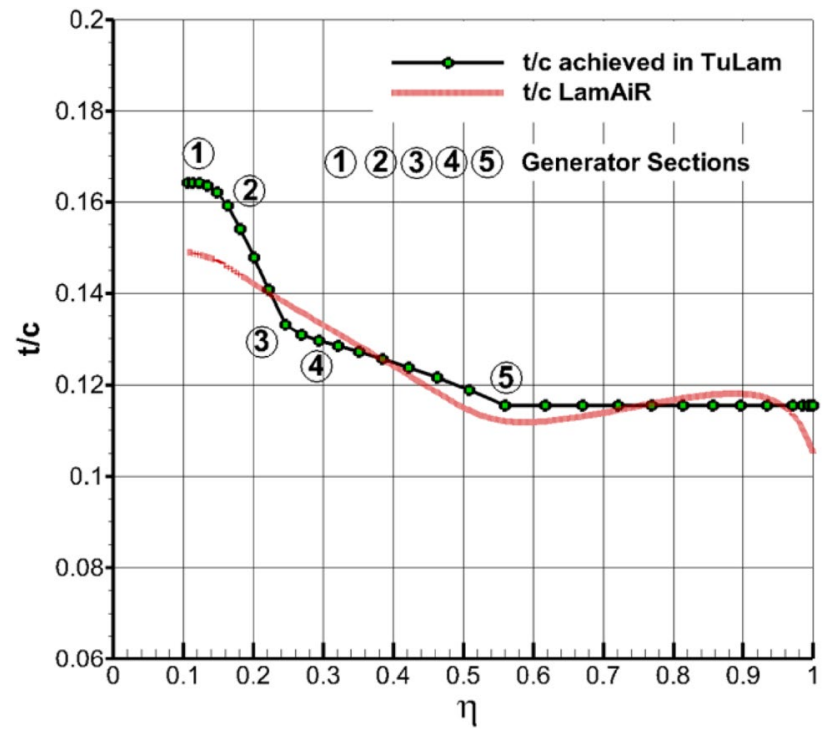

Fig. 9 Maximum relative thickness $t / c$ of the designed TuLam wing sections

$16 \%$ relative thickness at pos. 1) while the chordwise position of the maximum thickness in these sections is shifted to the rear. Figure 9 shows the spanwise distribution of relative thickness $\mathrm{t} / \mathrm{c}$ achieved in the TuLam wing design in comparison with the LamAiR results.

\section{First assessment of the design on overall aircraft level}

\subsection{Processing of the FLOWer results}

In order to perform a first assessment of the forward swept NLF wing on overall aircraft level, the FLOWer results were processed in a way that the following data were available: The spanwise distributions of maximum relative thickness $t / c$, local lift coefficient $c_{1}$ and circulation $c \times c_{1}$ as well as the dimensionless coordinates of the generated airfoil sections at span positions 1, 4 and 5 from Fig. 8 .

Additionally, the profile drag coefficient $C_{\mathrm{D} \text {,Prof }}$ (comprising friction drag, friction induced pressure drag and wave drag) of the wing was estimated, Fig. 10. For this purpose the wing was split into five segments and local profile drag coefficients representing the individual segment was calculated using the tool MSES-Runner, part of the MICADO preliminary design program. MICADO was developed by the Institut für Luft und Raumfahrt (ILR) of RWTH Aachen, [11]. It should be noted that MSES-Runner, like FLOWer $2.75 \mathrm{~d}$, takes into account sweep and taper. Subsequently, the local drag coefficient was weighted with the area of its corresponding segment, summed up and divided by the wing reference area of $122 \mathrm{~m}^{2}$. By this procedure the

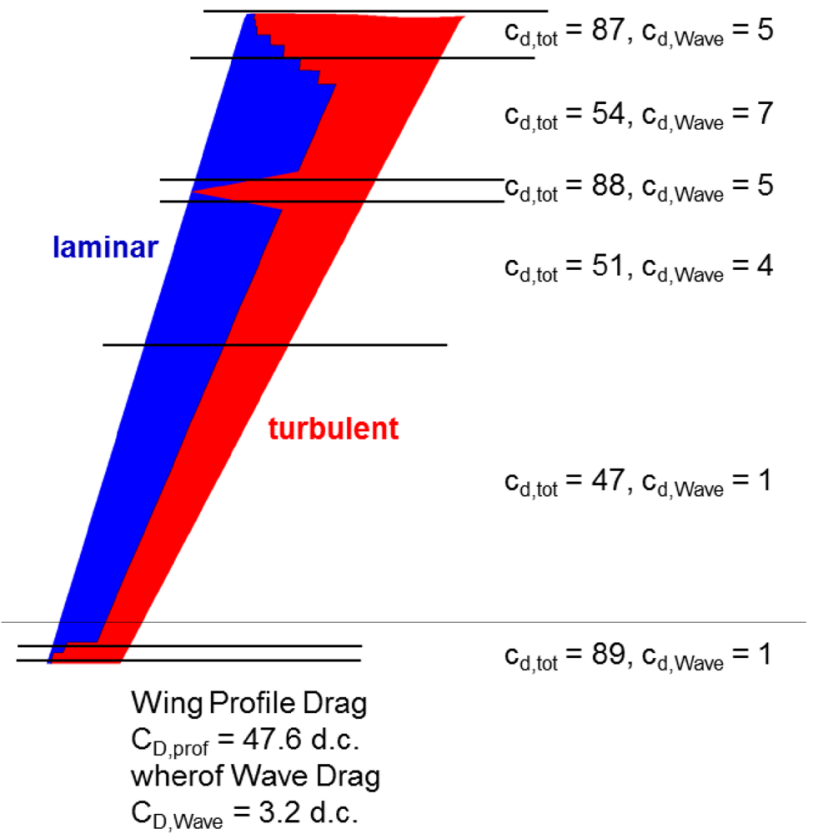

Fig. 10 Estimation of profile drag for the final wing design

profile drag coefficient of the wing was determined to be $C_{\mathrm{D}, \text { Prof,lam }}=48 \mathrm{dc}$ (it is noteworthy that the wetted area of the wing is roughly $100 \mathrm{~m}^{2}$ and therefore the total profile drag coefficient is less than its components). Hereby, the transition line shown in Fig. 10 was derived from a sectionwise stability analysis of the FLOWer $3 d$ flow solution, except the turbulent wedge in the vicinity of the engine pylon, which was assumed to have an opening angle of about $25^{\circ}$. In the same manner the profile drag of the TuLam wing in case of full chord turbulent flow was assessed resulting in $C_{\mathrm{D}, \text { Prof,turb }}=72 \mathrm{dc}$.

\subsection{Evaluation with MICADO}

For the evaluation of the TuLam wing design the aforementioned MICADO software was employed. For this purpose the aircraft geometry from the preliminary design stage with PrADO and from the detailed aerodynamic design (in particular the airfoil sections) were taken and a new aircraft sizing of the aircraft masses was performed for the design mission. A comparison of lift and circulation distribution between MICADO and FLOWer results show good agreement, Fig. 11.

In Fig. 12 the potential block fuel reductions of the TuLam aircraft in comparison with the reference CSR-01 are presented. The CSR-01 is essentially a re-design of the A320-200 obtained with the MICADO software, [12]. It can be seen that for the design mission of 2500NM the TuLam configuration already shows a block fuel reduction of $4 \%$ compared to CSR-01, even if the wing is full chord 
Fig. 11 Comparison of lift and circulation distribution from MICADO and FLOWer
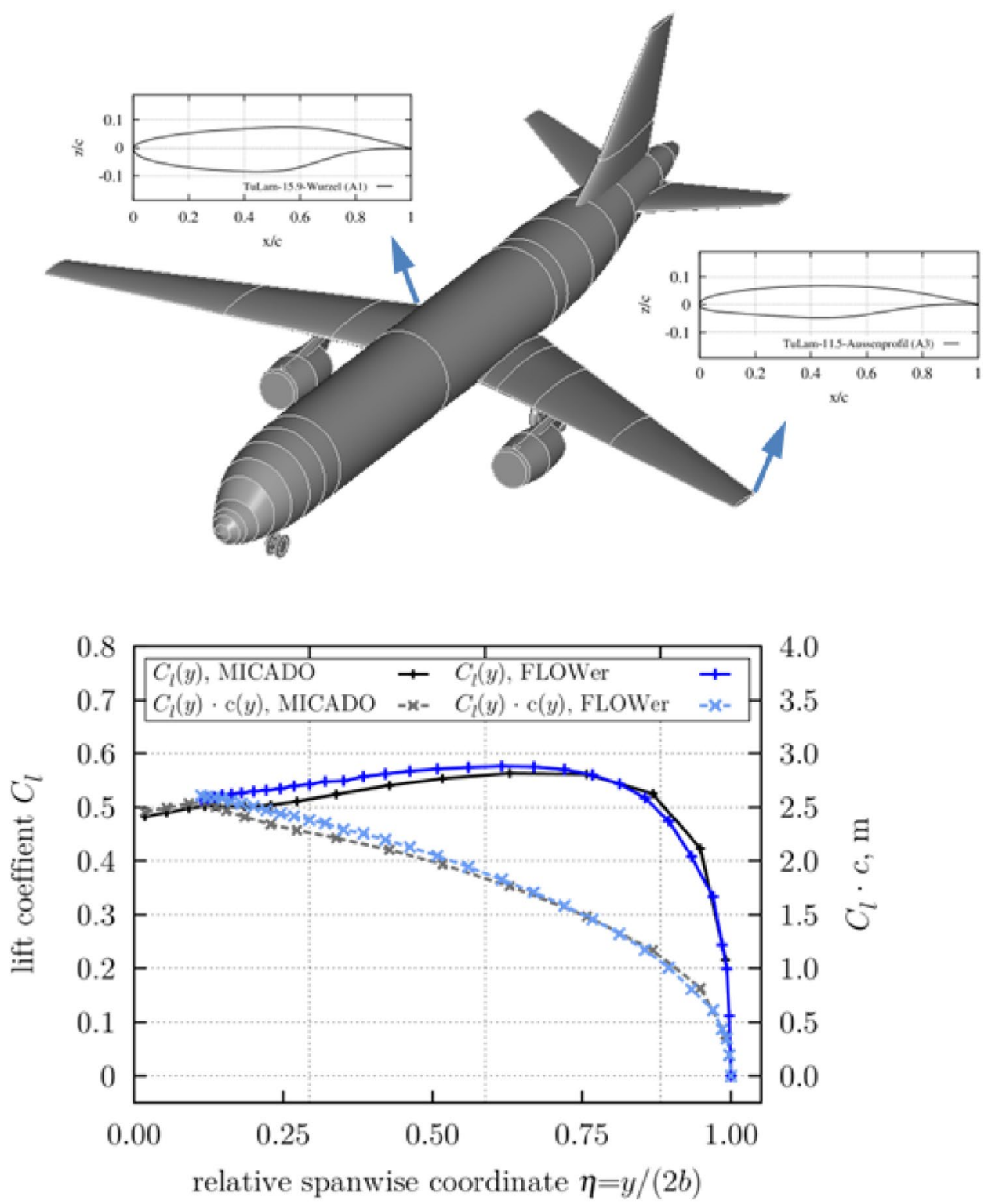

turbulent. The main reasons for this gain is a lighter wing resulting from comparatively thick airfoil sections (see Fig. 9; A320: $t / c$ at root ca. 0.145, at midspan ca.0.114, outboard.0.11) in conjunction with a spanwise load distribution of the mono trapezoidal forward swept wing that balances bending moment and induced drag in a favorable way.

From Fig. 12 one can also derive the beneficial effect of laminarization: As pointed out in Sect. 5.1, a maximum drag reduction of

$\triangle \mathrm{C}_{\mathrm{D}}=C_{\mathrm{D}, \text { Prof,turb }}-C_{\mathrm{D}, \text { Prof,lam }}=24 \mathrm{dc}$,

is feasible for the laminar TuLam wing compared to the full chord turbulent one. This delivers a block fuel reduction of almost exactly $12 \%$ for the design mission and slightly more than $8 \%$ for the shorter study mission (800NM with 150 Pax, no cargo). Of course, the shorter a mission is, the higher is the fuel mass fraction needed for flight in climb.

If the gradient of the curves in Fig. 12 is interpreted as a lever arm for further improvements due to the laminarization of other wetted components (like, for example, the fin from the HLFC technology path of the TuLam project) than a further block fuel reduction of up to $0.3 \%$ per dc drag reduction can be expected.

From a plot of the key aircraft characteristics, as shown in Fig. 13, the sources of block fuel reductions $\left(B F_{\mathrm{dm}}\right.$ for design mission and $B F_{\mathrm{sm}}$ for study mission) can be derived. Obviously, the main portion of the reduction comes from an improved $(L / D) o p t$, which, in turn, can mainly be contributed to the reduced profile drag by laminarization (with a minor share of wave drag reduction). However, the turbulent TuLam configuration also shows an improved (L/D)opt, 


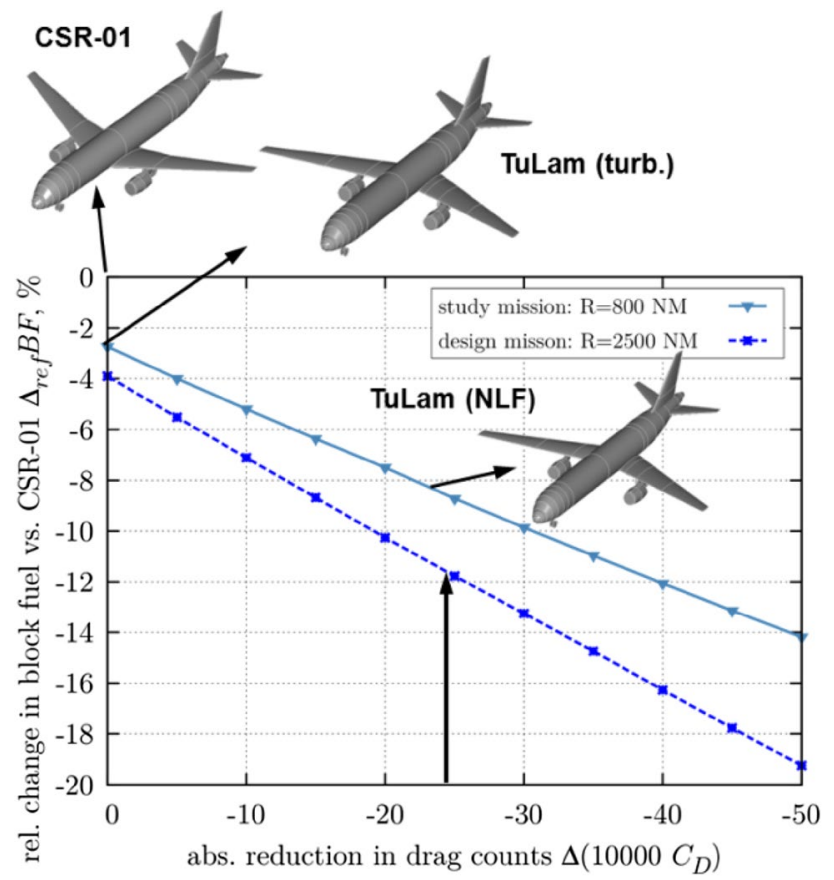

Fig. 12 Block fuel reduction for design mission and a shorter study mission as a function of profile drag reduction relative to full chord turbulent wing

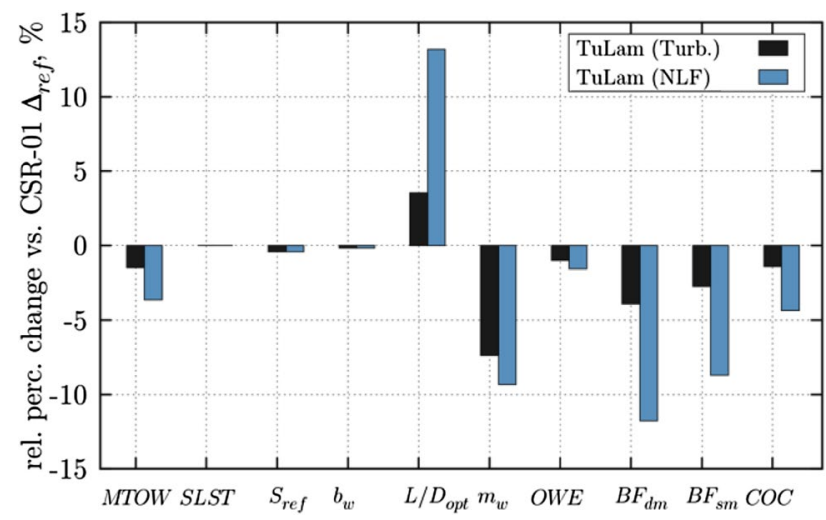

Fig. 13 Key aircraft characteristics of TuLam configuration (turbulent and laminar) in comparison with the CSR-01 reference

indicating that, with the profile drag being very similar to the reference aircraft, induced drag also was slightly lowered by the choice of planform and spanwise loading (it should be noted that wing reference area $S_{\text {ref }}$ and wing span $b w$ are marginally lower than for the reference, but the aspect ratio was held constant).

Another significant contribution to the $B F$ reduction comes from the lower wing mass $m_{w}$, made possible by the slightly over-elliptic inboard loading and relatively thick airfoil sections close to the wing root. Due to mass-snowball-effects, less fuel weight leads to a reduced MTOW (Maximum Take-Off Weight) and therefore less overall strain, the laminar TuLam aircraft has a slightly lighter wing mass $m_{w}$ and $O W E$ (Operating Weight Empty) than the turbulent one.

It should be emphasized that the Sea Level Static Thrust (SLST) shows no difference between Tulam and CSR01, i.e. no re-sizing of the engine was performed, so block fuel reductions can exclusively be traced back to the forward swept NLF wing concept and the accompanying changes in structural weight.

The last parameter shown in Fig. 13, COC, denotes the Cash Operating Costs. From an engineering point of view rather unimportant, it is decisive from a commercial perspective. However, since cost calculations strongly depend on the different views of operators on this subject, only a rough estimate can be given.

\section{Summary and outlook}

Within the frame of the DLR project TuLam a short and medium range transport aircraft with forward swept laminar wing was designed matching the top level aircraft requirements of the A320-200. The detailed aerodynamic design showed that natural laminar flow is feasible also at a design Mach number of $\mathrm{Ma}=0.78$ (and off design conditions up to $\mathrm{Ma}=0.80$ ). This feature distinguishes the TuLam configuration from those NLF designs with backward swept wings, where the combination of Reynolds number and necessary high leading edge sweep to counteract compressibility effects limits the cruise Mach number range to $\mathrm{Ma}=0.75$. As an additional feature, a $10 \%$ chord trailing edge flap was investigated that allows for robust laminar flow throughout the whole cruise flight domain.

A first evaluation on basis of the generated data from a detailed aerodynamic wing design shows a potential block fuel reduction in the order of $12 \%$ for the design mission (but less for shorter missions, when climb flight starts dominating fuel consumption). It is planned for the near future to enhance the validity of these first positive results by providing aerodynamic key figures that are more precise. Therefore, a complex geometry model of the TuLam configuration comprising wing, body with belly-fairing, pylon and engine has already been generated as a basis for the analysis with the "high-fidelity" CFD method Tau. In this context, off design trailing edge flap deflections will be optimized. Finally it should be noted that for the aforementioned complex geometry a high lift system as defined in Sect. 2.3 has already been designed and assessed with the Tau flow solver delivering an aircraft $C_{\mathrm{L}, \max }=2.85$, [13]. 
Open Access This article is distributed under the terms of the Creative Commons Attribution 4.0 International License (http://creativeco mmons.org/licenses/by/4.0/), which permits unrestricted use, distribution, and reproduction in any medium, provided you give appropriate credit to the original author(s) and the source, provide a link to the Creative Commons license, and indicate if changes were made.

\section{References}

1. Seitz, A., Kruse, M., Wunderlich, T., Bold, J., Heinrich, L.: The DLR project LamAiR: design of a NLF forward swept wing for short and medium range transport application. In 29th AIAA Applied Aerodynamics Conference, Hawaii, USA (2011)

2. Redeker, G., Horstmann, K.H., Quast, A., Dressler U., Bieler, H.: Flight tests with a natural laminar glove on a transport aircraft. In: AIAA Paper 90-30444 (1990)

3. Seitz, A., Horstmann, K.H.: Design studies on NLF and HLFC applications at DLR. In: international council of the aeronautical sciences, ICAS Konferenz, Nizza (2010)

4. Horn, M., Seitz, A., Schneider, M.: Novel tailored skin single duct concept for HLFC fin application. In: 2017-44, 7th European Conference for Aeronautics and Space Sciences (EUCASS), Mailand (2017). https://doi.org/10.13009/eucass

5. Piening, M.: Die statische Aeroelastizität des anisotropen Tragflügels. In: DGLR Symposium Entwicklung und Anwendung von CFK-Strukturen, Bericht 84-02, Berlin (1984)

6. Kruse, M., Wunderlich, T., Heinrich, L.: A conceptual study of a transonic NLF transport aircraft with forward swept wings. In: 30th AIAA Applied Aerodynamics Conference, New Orleans, USA (2012)
7. Strüber, H., Wild, J.: Aerodynamic design of a high-lift system compatible with a natural laminar flow wing within the DeSiReH project. In: 29th Congress of the International Council of the Aeronautical Sciences, St. Petersburg (2014)

8. Heinze, W.: PrADO; Ein Beitrag zur quantitativen Analyse der technischen und wirtschaftlichen Auslegungsgrenzen verschiedener Flugzeugkonzepte für den Transport großer Nutzlasten. In: ZLR-Forschungsbericht 94-01, Dissertation Technische Universität Braunschweig, ISBN 3-928628-14-3 (1994)

9. Streit, T., Hofrogge, N.: DLR transonic inverse design code, extensions and modifications to increase versatility and robustness. In: RAeS 2016 Appllied Aerodynamics Conference, Bristol (2016)

10. Eppler, R.: Airfoil design and data. Springer Verlag GmbH, Heidelberg (1990)

11. Risse, K., Anton, E., Franz K., Hörnschmeyer, R.: An integrated environment for preliminary aircraft design and optimization. In: 8th AIAA Multidisciplinary Design Optimization Specialist Conference, Hawaii, USA (2012)

12. Risse, K., Schäfer, K., Schültke, F., Stumpf, E.: Central Reference Aircraft data System (CeRAS) for research community. CEAS Aeronaut. J. 7(1), 121-133 (2016)

13. Keller, D.: High-lift design for a forward swept natural laminar flow wing. In: Deutscher Luft- und Raumfahrtkongress 2017, Garching bei München (2017)

Publisher's Note Springer Nature remains neutral with regard to jurisdictional claims in published maps and institutional affiliations. 